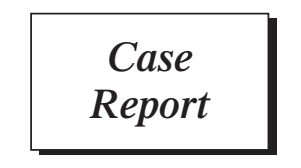

\title{
Coexisting Thymic and Pulmonary Carcinoid Tumors Associated with Multiple Endocrine Neoplasia Type1
}

\author{
Makoto Motoishi, MD, ${ }^{1}$ Kanna Horimoto, MD, ${ }^{1}$ Kazuki Hayashi, MD, ${ }^{1}$ \\ Satoru Sawai, MD, $\mathrm{PhD},{ }^{1}$ and Koki Moriyoshi, $\mathrm{MD}, \mathrm{PhD}^{2}$
}

\begin{abstract}
An anterior mediastinal tumor was detected in a 45-year-old female during a medical checkup. Chest computed tomography (CT) showed the anterior mediastinal tumor and a pulmonary tumor in the right lower lobe. Furthermore, tumors of the parathyroid gland, pancreas, and pituitary gland were also detected. She was clinically diagnosed with multiple endocrine neoplasia type1 (MEN1). The patient underwent extended thymectomy combined with mediastinal lymph node dissection and wedge resection of the lung including the right pulmonary lesion via a median sternotomy. We diagnosed the patient with an atypical carcinoid tumor of the thymus, a typical pulmonary carcinoid tumor.
\end{abstract}

Keywords: multiple endocrine neoplasia type1, thymic carcinoid tumor, pulmonary carcinoid tumor

\section{Introduction}

Multiple endocrine neoplasia type1 (MEN1) is an autosomal dominant disease characterized by neoplasia in the parathyroid gland, anterior pituitary gland, endocrine pancreas, and duodenum. In addition, carcinoid tumors (thymic, gastric, and bronchopulmonary tumors) have been reported to develop in MEN1. The first case of a thymic carcinoid tumor associated with MEN1 was described by Rosai et al. ${ }^{1)}$ in 1972. Although little is known about the condition, it is reported that most

${ }^{1}$ Department of Thoracic Surgery, National Hospital Organization Kyoto Medical Center, Kyoto, Kyoto, Japan

${ }^{2}$ Department of Pathology, National Hospital Organization Kyoto Medical Center, Kyoto, Kyoto, Japan

Received: February 20, 2017; Accepted: July 13, 2017

Corresponding author: Makoto Motoishi, MD. Department of Thoracic Surgery, National Hospital Organization Kyoto Medical Center, Fukakusamukaihata-cho, Fushimi-ku, Kyoto, Kyoto 612-8555, Japan

Email: mmotoish@kyotolan.hosp.go.jp

(C)2018 The Editorial Committee of Annals of Thoracic and Cardiovascular Surgery. This work is licensed under a Creative Commons Attribution-NonCommercial-NonDerivatives International License. patients with MEN1 and thymic carcinoid tumors die of their thymic carcinoid tumors. ${ }^{2)}$ In contrast, bronchopulmonary carcinoid tumors associated with MEN1 do not appear to result in a poor prognosis in the majority of patients. ${ }^{3)}$ Herein, we describe the first reported case of coexisting thymic and pulmonary carcinoid tumors associated with MEN1.

\section{Case Report}

An anterior mediastinal tumor was detected in a 45year-old female with dyspnea on effort during a medical checkup. Chest computed tomography (CT) showed the anterior mediastinal tumor and a pulmonary tumor in the right lower lobe. Furthermore, tumors of the parathyroid gland, pancreas, and pituitary gland were also detected. She was clinically diagnosed with MEN1 and was referred to our hospital for further investigation and treatment. She had never smoked. She had a history of renal stones, hypercalcemia, and hyperparathyroidism, for which she underwent parathyroidectomy of the right upper and left lower glands at the age of 35 years old. Her father had a history of hyperparathyroidism, pancreatic insulinoma, and a duodenal carcinoid tumor, 

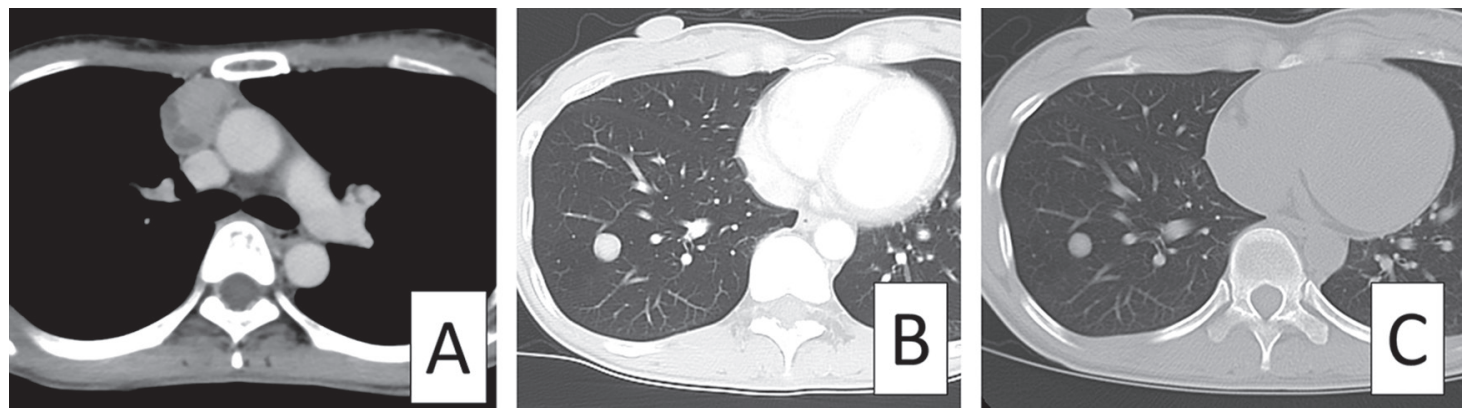

Fig. 1 (A-C) Chest CT revealed an anterior mediastinal tumor (maximum dimension: $4 \mathrm{~cm})(\mathbf{A})$ and a pulmonary tumor in the right lower lobe (diameter: $1.3 \mathrm{~cm})(\mathbf{B})$. A chest CT scan taken 8 years ago had shown a pulmonary tumor $(\mathbf{C})$. CT: computed tomography
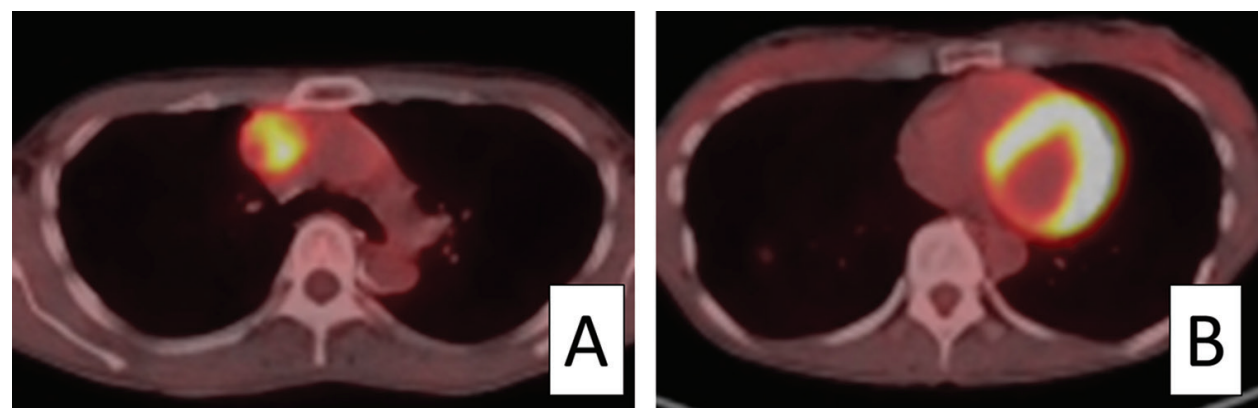

Fig. 2 (A and B) PET-CT showed abnormal accumulation in the thymic lesion (A), but no significant uptake was observed in the pulmonary lesion (B). PET: positron emission tomography; CT: computed tomography

but had not been diagnosed with MEN1. As was found at the previous institution, chest CT revealed an anterior mediastinal tumor (maximum dimension: $4 \mathrm{~cm}$ ) (Fig. 1A) and a pulmonary tumor in the right lower lobe (diameter: $1.3 \mathrm{~cm}$ ) (Fig. 1B). Compared with 8 years ago (when it was first detected) (Fig. 1C), the pulmonary lesion had enlarged slightly, but no anterior mediastinal tumor was noted when the pulmonary lesion had first been diagnosed. Cervical ultrasonography also demonstrated parathyroid tumors of the right lower and left upper glands. Abdominal CT and brain magnetic resonance imaging showed pancreatic, duodenal, and pituitary tumors. Preoperative positron emission tomography (PET)-CT detected strong accumulation in the thymic lesion (maximum standardized uptake value: 6.97) (Fig. 2A), but no significant uptake was observed in the pulmonary lesion (Fig. 2B). Laboratory tests demonstrated elevated serum levels of calcium $(10.6 \mathrm{mg} / \mathrm{dL}$, normal range: $8.7-10.3 \mathrm{mg} / \mathrm{dL})$, intact parathyroid hormone $(82 \mathrm{pg} / \mathrm{mL}, 10-65 \mathrm{pg} / \mathrm{mL})$, thyroid-stimulating hormone (TSH) $(4.951 \mu \mathrm{IU} / \mathrm{mL}$, $0.350-4.940 \mu \mathrm{IU} / \mathrm{ml})$, free triiodothyronine $(4.28 \mathrm{pg} / \mathrm{mL}$, $1.71-3.71 \mathrm{pg} / \mathrm{mL})$, prolactin $(51.5 \mathrm{ng} / \mathrm{mL}, 5-30 \mathrm{ng} / \mathrm{mL})$, and gastrin (362 pg/mL, 37-172 pg/mL). The levels of other hormones, such as insulin, adrenocorticotropic hormone, growth hormone, and cortisol were within normal limits.

The patient underwent extended thymectomy combined with mediastinal lymph node dissection and wedge resection of the lung including the right pulmonary lesion via a median sternotomy. A swollen lymph node about $5 \mathrm{~mm}$ was detected in contact with the main tumor during operation. Right lower parathyroidectomy was performed simultaneously. A histopathological examination of the thymic lesion involving hematoxylin-eosin staining revealed oval and round tumor cells with eosinophilic cytoplasm and oval nuclei, which exhibited a nested growth pattern. Three mitoses/10 high-power field (HPF) were detected without apparent necrosis (Fig. 3A). In the pulmonary lesion, tumor cells with eosinophilic cytoplasm and oval nuclei displayed glandular, trabecular, and nested growth patterns. No mitosis or necrosis was detected (Fig. 3B). During immunohistochemistry, both the thymic and pulmonary tumors were found to be positive for synaptophysin (Figs. 3C and 3D) and chromogranin A. The Ki-67 


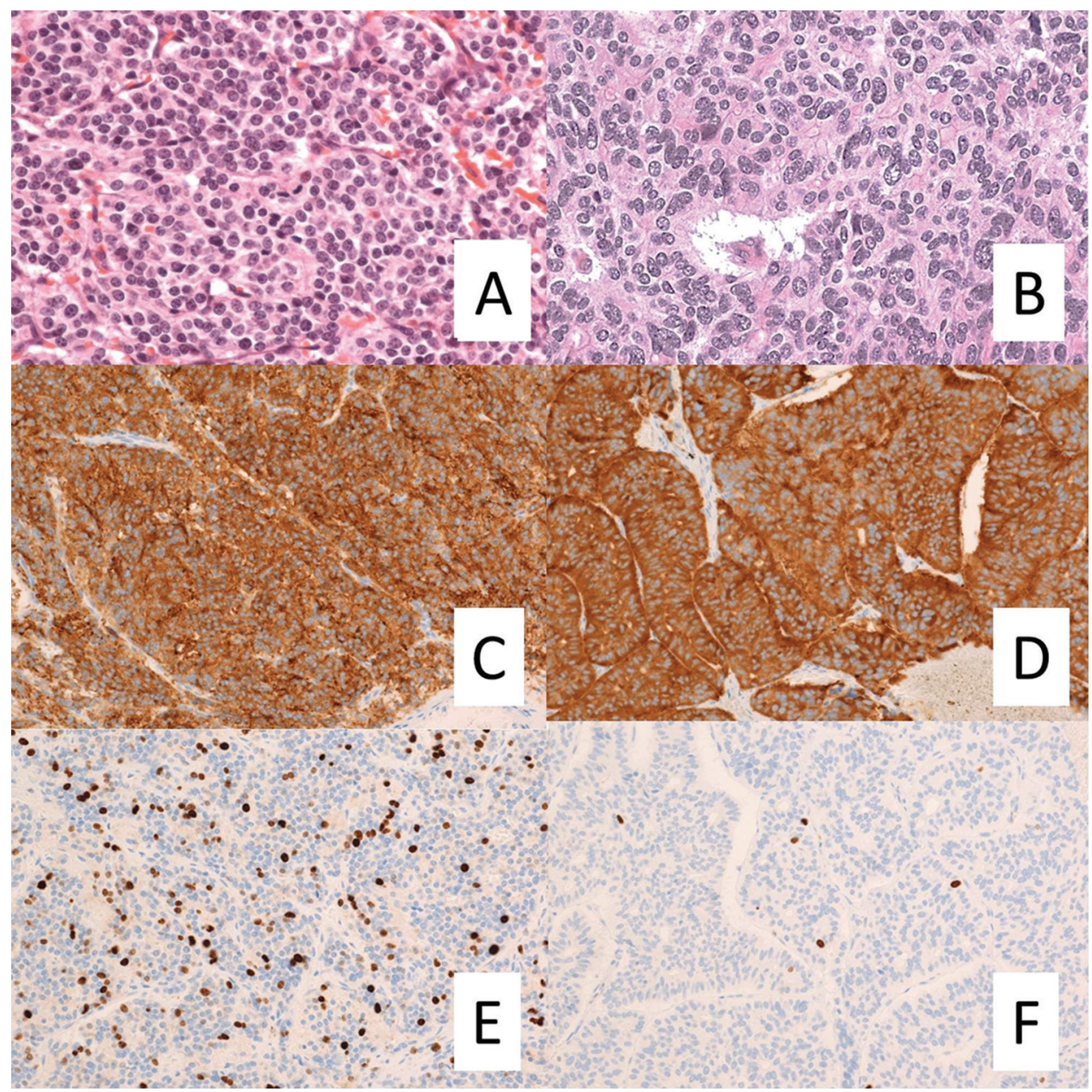

Fig. 3 (A-F) A histopathological examination of the thymic lesion (A) hematoxylin-eosin staining, (C) synaptophysin, E: Ki67 and the pulmonary lesion (B) hematoxylin-eosin staining, (D) synaptophysin, (F) Ki67.

labeling indices of the thymic (Fig. 3E) and pulmonary lesions (Fig. 3F) were $11 \%$ and $<1 \%$, respectively. We diagnosed the patient with an atypical carcinoid tumor of the thymus, which had metastasized to the anterior mediastinal lymph nodes; a typical pulmonary carcinoid tumor; and hyperplasia of the parathyroid gland. Transient hypocalcemia was seen postoperatively, but it subsided spontaneously. After the operation, the patient received radiotherapy involving a total dose of $54 \mathrm{~Gy}$ to her mediastinum. Subsequently, operations for abdominal and cranial lesions were performed in order. The patient diagnosed as insulinoma and glucagonoma of the pancreas, gastrinoma and somatostatinoma of the duodenum, and TSH-secreting adenoma of the pituitary gland, respectively. She was free from clinically obvious recurrence 2 years after the operation for her chest lesions.

\section{Discussion}

Thymic carcinoid tumors associated with MEN1 are rare. They have been reported to exhibit prevalence rates of $8 \%,{ }^{4)} 4.9 \%,{ }^{5)}$ and $3.1 \%$ in the literature. Thymic carcinoid tumors are associated with smoking and male gender, and the majority of them develop between the third and fifth decades. ${ }^{2,4-6)}$ On the other hand, Sachithanandan et al. reported that bronchopulmonary carcinoid tumors occur in at least $5 \%$ and up to $31 \%$ of adults with MEN1, and are more common in females than in males. ${ }^{3)}$ In our case, the pulmonary lesion was detected 8 years before the anterior mediastinal tumor, and it had enlarged slightly in the intervening period. The thymic and pulmonary lesions were diagnosed as atypical and typical carcinoid tumors, respectively. Furthermore, she was free from recurrence 2 years after 
the operation without any systemic adjuvant therapy. According to her clinical course and histological findings, we diagnosed the patient with coexisting thymic atypical carcinoid and pulmonary typical carcinoid tumors. To the best of our knowledge, this is the first reported case of coexisting thymic and pulmonary carcinoid tumors associated with MEN1.

MEN1-related hyperparathyroidism is the most common (it is seen in $>95 \%$ of MEN1 patients) (and usually the first) manifestation of MEN1.7) Unfortunately, our patient was not suspected of accompanying MEN1 when she was diagnosed with hyperparathyroidism in spite of her family history and the fact that the pulmonary lesion was suspected to be a carcinoid tumor. Instead, a thymic carcinoid tumor was detected during a medical checkup, and the patient was diagnosed with advanced disease. Gibril et al. ${ }^{4)}$ stated that thymic carcinoid tumors are generally a late manifestation of MEN1, and they did not occur as the initial manifestation of MEN1 in any of their patients. In the current case, it might have been possible to detect the thymic carcinoid tumor at an early stage if the patient had been diagnosed with MEN1 earlier and followed-up regularly.

Thymic carcinoid tumors associated with MEN1 often result in death. On the contrary, bronchopulmonary carcinoid tumors do not appear to exhibit a poor prognosis in the majority of patients. ${ }^{3)}$ Therefore, the management of thymic carcinoid tumors is more important than the management of bronchopulmonary carcinoid tumors. To detect thymic carcinoid tumors as early as possible, yearly chest CT scans are recommended for screening purposes in all patients with MEN1 who are older than 25 years of age, particularly in males. ${ }^{4)}$ In addition, it is recommended that prophylactic thymectomy should be considered during the course of neck exploration for parathyroidectomy, particularly in male smokers. ${ }^{6}$

\section{Conclusion}

We report the first case of coexisting thymic and pulmonary carcinoid tumors associated with MEN1. Clinicians should be aware that thymic carcinoid tumors can develop in patients with MEN1-related pulmonary carcinoid tumors.

\section{Disclosure Statement}

The authors have no conflicts of interest to declare.

\section{References}

1) Rosai J, Higa E, Davie J. Mediastinal endocrine neoplasm in patients with multiple endocrine adenomatosis. A previously unrecognized association. Cancer 1972; 29: 1075-83.

2) Sugiura H, Morikawa T, Itoh K, et al. Thymic carcinoid in a patient with multiple endocrine neoplasia type 1: report of a case. Surg Today 2001; 31: 428-32.

3) Sachithanandan N, Harle RA, Burgess JR. Bronchopulmonary carcinoid in multiple endocrine neoplasia type 1. Cancer 2005; 103: 509-15.

4) Gibril F, Chen YJ, Schrump DS, et al. Prospective study of thymic carcinoids in patients with multiple endocrine neoplasia type 1. J Clin Endocrinol Metab 2003; 88: 1066-81.

5) Teh BT, McArdle J, Chan SP, et al. Clinicopathologic studies of thymic carcinoids in multiple endocrine neoplasia type 1. Medicine (Baltimore) 1997; 76: 21-9.

6) Ferolla P, Falchetti A, Filosso P, et al. Thymic neuroendocrine carcinoma (carcinoid) in multiple endocrine neoplasia type 1 syndrome: the Italian series. J Clin Endocrinol Metab 2005; 90: 2603-9.

7) Teh BT, Zedenius J, Kytölä S, et al. Thymic carcinoids in multiple endocrine neoplasia type 1. Ann Surg 1998; 228: 99-105. 